\title{
OBJECT COMPOSITION: A CASE STUDY
}

\author{
Dunia Ramazani, Discreet Logic \\ Gregor v. Bochmann, University of Ottawa
}

\begin{abstract}
In [10], we have presented an object-oriented method, called eXtended Object Modelling Technique, XOMT for shorthand. In this paper, we modify the developmental approach used in XOMT to include the specification of the behavior of composite objects based on synchronous interactions. The new developmental process consists of describing the application structure in terms of objects and associations between these objects. Object associations are then further refined by describing the object interactions that occur in the context of these associations. Object and association behaviors are specified in CSL, a specification language based on rendezvous interaction. CSL specifications can be translated in TLA. This adds a reasoning capability to the development process. The translation into TLA is motivated by the existence of a variety of specification and verification tools for TLA. Keywords: Case study, CSL, Formal reasoning, Object composition, OMT, TLA, XOMT.
\end{abstract}

\section{Introduction}

In software engineering, experimentation is a necessary adjunct to process improvement. Objective and meaningful case studies can help us understand particular object-oriented notations, methods, or languages. In [10], the presentation of eXtended Object Modelling Technique, XOMT for shorthand was aimed at presenting the concepts and principles underlying XOMT. Now we have designed a new specification language for capturing the behavior of composite objects, namely CSL which stands for Composition Specification Language. This paper shows how these latter results can be combined with XOMT. A specification case study is used for conveying our ideas, and demonstrating the usability of the results in the context of XOMT.

The integration of CSL is achieved at the expense of modifying the developmental approach used in XOMT. We departed from the OMT-based [12] development process to a lighter process based on describing the application and object structures in terms of objects and associations between these objects. Object associations are then further refined by describing the object interactions that occur in the context of these associations. Object behaviors and association behaviors are specified in CSL, which can be translated into TLA [6]. This adds a reasoning capability to the development 
process, i.e. we may then use TLA tools for verifying the specifications. The application selected in this case study is the specification of a lift system.

The paper is structured as follows. In Section 2, we begin by reviewing the essential concepts and principles of XOMT. It is followed by a summary of features provided by CSL including its formal semantics based on its translation into TLA. We then summarize the development process which uses CSL. In Section 3, the development process is put in practice through the specification of a lift system. This includes the description of the application requirements, the informal specification supported by XOMT and the specifications in CSL, including their translation into TLA and a demonstration of some formal reasoning. We close the paper with the lessons learned in this experiment and point to some future work.

\section{Overview of XOMT and CSL}

\subsection{Concepts and Principles underlying XOMT}

Roughly speaking, XOMT is OMT with a few add-ons allowing to specify composite objects such that there is a linkage between the structure and the behavior of these objects. In XOMT, properties of composite objects are classified into inherent, aggregate, and emergent properties. A composite object is an object with an internal structure which consists of the components, and the interconnections including the dynamic interactions between the components of the composite object. The linkage between component properties and composite object properties is established by distinguishing inherent properties, i.e. properties of the composite object which semantics is provided by properties of its components, from aggregate properties, properties of the composite object obtained by combining the properties of its components using aggregation mechanisms, and from emergent properties which are properties of the composite object which do not depend on component properties.

This new classification requires notational changes to object models in order to capture visibility and hiding of components, promotion of component properties to the status of composite object properties, and aggregation mechanisms used to combine the component properties. Other areas of improvement of OMT include the specification of the communication between objects, especially the communication between the components of a given composition. A more abstract interaction mechanism based on behavior constrainment is proposed, namely Contract specifications where behavioral interactions are expressed in a more abstract way so that the description does not introduce implementation bias. It is achieved by constraining, through predicates, the behavior of the interacting objects. Contracts are related to object associations abstracting the interactions between the classes. They represent the concurrent composition of the participant statecharts (individual behaviors) such that the behavior of the participants conforms to the constraints explicitly stated in the contracts. Constraints are expressed using a combination of first order predicate logic and OMT constraints.

\subsection{Steps and notation in XOMT}

The description of composite objects proceeds in three steps [9]. The configuration describes the structure of the composite in terms of components, associations and behavioral interactions among these. The next step, juxtaposition shows how the composite is linked to its components through the aggregation association, and 
inherent and aggregate properties. The last step, emergence concerns the specification of emergent properties.

Aggregation association is represented like in OMT. Visibility/hiding of components is specified by having the component class represented respectively with doubled and simple framed rectangles. The attributes and operations of composite are classified using three separate rectangles. Inherent, aggregate, and emergent attributes and operations are represented using respectively inherent, aggregate, and emergent rectangles, that is three rectangles in place of one, like for classes in OMT. Inherent associations are of two kinds, those resulting from the visibility of components and those involving hidden components. The former are represented by associations crossing the boundary of the composite and ending at some visible component inside the composite. The latter are represented by associations ending at the boundary of the composite and continued within the composite by a dashed line to some hidden component. Aggregate and emergent associations are represented using associations ending at the boundary of the composite and respectively decorated with the annotations $\{\mathrm{A}\}$ and $\{\mathrm{E}\}$.

To describe the behavior, we use parallel composition of statecharts. This is represented by having the parallel statecharts separated by a dashed line while being enclosed in the composite statechart [3]. Inherent behavior is represented by the statechart of visible components and a specific statechart (annotated with "promoted") for the behavior originating from hidden components. Aggregate and emergent behaviors are respectively specified using specific statecharts. The overall behavior of the composite object consists of parallel composition of inherent, aggregate, and emergent behavior.

\subsection{Composition Specification Language (CSL)}

2.3.1. Concepts and principles. CSL has its roots in the assumption that object interactions occur only over object associations. In CSL, object interactions are called abstract events [2] and they correspond to joint-actions [4,5]. They are characterized by:

(a) There is no asymmetric caller/callee relationship: It is not said which object makes the decision for the execution of an interaction.

(b) There may be more than two objects participating in a given interaction, i.e. multiparty interactions.

(c) Each participating object may impose certain conditions which must be satisfied when the interaction occurs. Each participating object may also define some local state changes that occur during the execution of the interaction.

Once we are able to describe the interactions that occur in the context of object associations, we may use the same approach for describing the interactions between components in a composition. Using this approach, we are able to describe structures of compositions. Composition in CSL is achieved by connecting the objects and then hiding certain abstract events which involve these objects. Hiding is used as an abstraction mechanism.

2.3.2. Notation. The notation proposed by CSL is portrayed below. In CSL, we assume that a simple object (non-composite) can be represented as a composite object which has no components. Based on that assumption, we use a single template for specifying objects. Only object compositions have the "inherits" section which indicates the actions of the components which are also actions of the composition. 


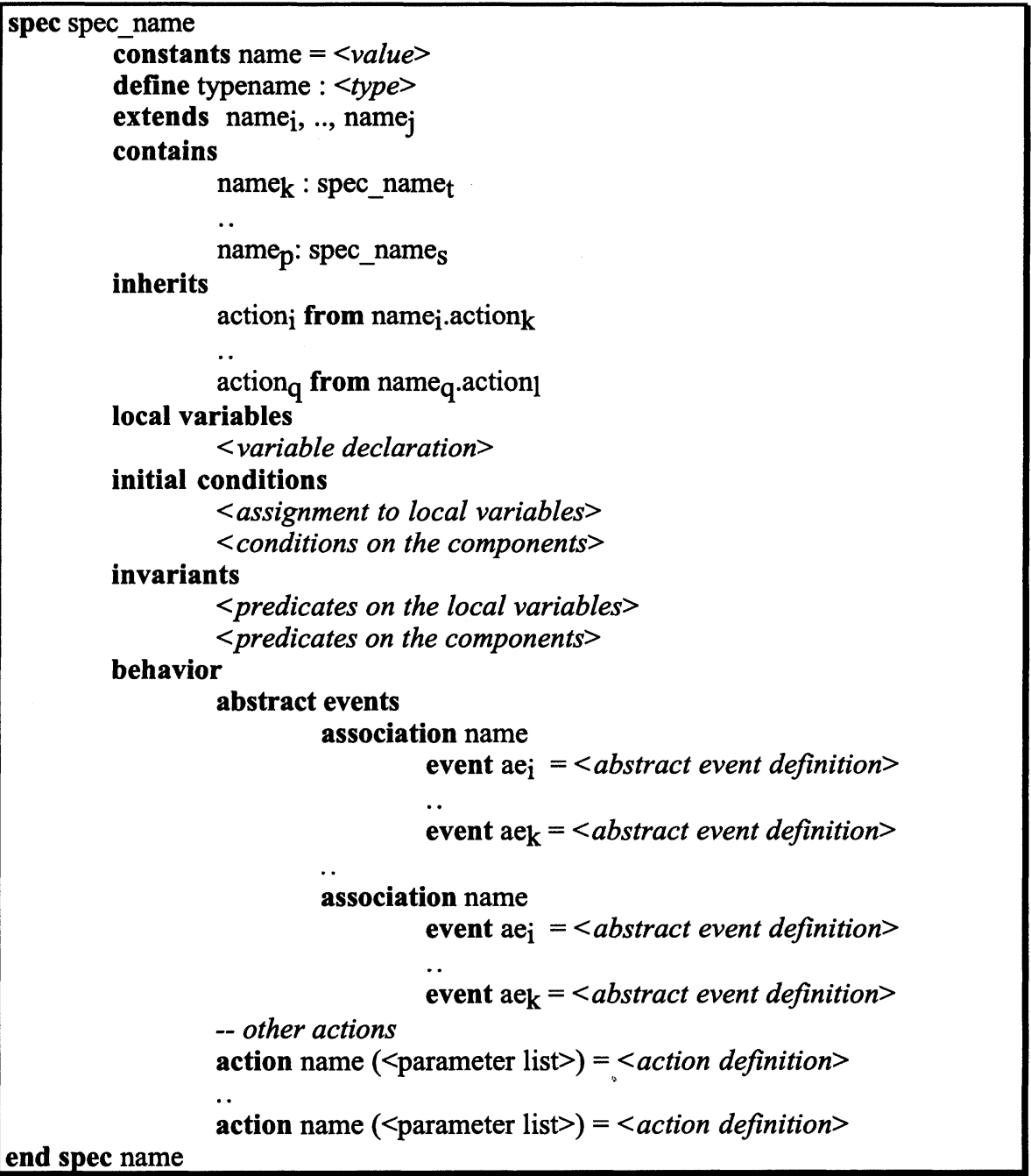

Figure 1. Object template.

The "constants" and "define" clauses are self-explanatory. They introduce constants and new types in the specification. The "extends" clause denotes specialization. Features of the specifications listed in the "extends" clause are augmented with new features introduced by the specification. Actions can be redefined, invariants more constrained, etc.

The "contains" clause indicates the structure of the composition. It lists the components, while the constraints on these components can be stated in the "invariants" clause. "abstract events" denote the abstract events occurring between the components. These abstract events are structured according to the associations between the objects inside the composition. "local variables" denote the states of the object or the composition. The clause "initial conditions" defines the initial state. It assigns the initial values to the local variables and may define the initial states of the 
components in the context of the composition. "invariants" are used to record the safety properties of the object, and its components.

The "behavior" clause defines the actions which are supported by the object. Actions may have parameters. The semantics of the behavior clause are: First the initial conditions are established, in any state either an action occurs, or the local variables remain unchanged. The invariants must be always satisfied in the state before the execution of the action and in the state after the execution of the action. At the level of an object, we assume interleaving concurrency between the actions supported by that object. For a composition, there is interleaving between its abstract events, and its actions.

Until now we have not said too much about actions. Actions are defined in terms of "enabled", "defined", and "changes" predicates. These predicates involve the local variables of the specification. In action definitions, the "defined" predicate indicates the hypotheses about the environment for the action. In particular, the "changes" predicate will normally relate the new values of parameters and local variables to the values before the execution of the action. We use a combination of mathematics and predicate logic to define the semantics of actions. The meaning of an action is:

(a) when "enabled" is satisfied and "defined" is also satisfied, then the action can be executed and "changes" will be true afterwards;

(b) when "enabled" is satisfied and "defined" is not satisfied, the action can be executed, but the result is undefined;

(c) when "enabled" is not satisfied, the action can not be executed.

An abstract event is the synchronization of two or more actions. It is specified by a list of actions which are synchronized and a constraint over the parameters of the synchronized actions. The abstract event is enabled in states where all its composing actions are enabled and the constraint is true. The defined clause of one action involved in an abstract event should be consistent with the definition of the other actions involved in the abstract event, i.e. there are not values of the variables appearing in the defined predicate which are such that the defined predicate is false and the enabled predicates of the actions involved in the abstract event are true. This is referred to as internal consistency of the definition of an interaction.

The specification parameters, local variables, actions, and abstract events are by default visible unless tagged with the keyword hidden.

2.3.3. Formal semantics. To reason about CSL specifications, we need an underlying execution model as well as an underlying logic for proving properties. We have favored the translation of CSL specifications into TLA [6], an existing formal specification language. The choice of TLA (Temporal Logic of Actions) is motivated by its computational model which is based on interaction, its built-in notion of behavior including the temporal ordering of actions, and nonetheless the availability of a wide range of specification and verification tools [7].

The main difference between CSL and TLA is on the semantics of actions. CSL actions have explicit parameters. In addition, each CSL action indicates what are its assumptions about the environment since the objects are composed through their actions. This semantics introduce possible undefined behavior for actions. The action also has an explicit enabling condition.

When translated into TLA, CSL action parameters are introduced as additional variables of the TLA specification. In order to represent, in TLA, the case where the "defined" predicate of an action is not satisfied, we take the following approach for translating the action semantics. We write $L V\left(a_{i}\right)$ for the set of local variables appearing in the CSL action $a_{i}, V$ for the set of all local variables of the entire CSL 
specification. $\mathrm{CH}\left(\mathrm{a}_{\mathrm{i}}\right)$ is a subset of $\operatorname{LV}\left(\mathrm{a}_{\mathrm{i}}\right)$; it denotes the local variables which are modified by the action $a_{i} . \operatorname{PAR}\left(a_{i}\right)$ is the set of the parameters of the action $a_{i}$. Parameters which do not appear primed in the "changes" predicate of the action can be considered as input parameters. The others are output parameters. Based on this observation, $\operatorname{PAR}\left(a_{i}\right)$ can be decomposed into two sets $\operatorname{PAR}_{i n}\left(a_{i}\right)$ for input parameters and $\operatorname{PAR}_{\text {out }}\left(\mathrm{a}_{i}\right)$ for output parameters. Recall that a CSL action is defined by three predicates, namely enabled, defined, changes which will be respectively referred to in the sequel by $a_{i}$.enabled, $a_{i}$.defined, $a_{i}$.changes. The translation of an CSL action $a_{i}$ into TLA is defined as follows:

$$
\begin{aligned}
\forall \mathrm{LV}\left(\mathrm{a}_{\mathrm{i}}\right): \forall \operatorname{PAR}\left(\mathrm{a}_{\mathrm{i}}\right): & \wedge \mathrm{a}_{\mathrm{i}} \cdot \text { enabled } \\
& \wedge\left(\mathrm{a}_{\mathrm{i}} \cdot \text { defined } \Rightarrow \mathrm{a}_{\mathrm{i}} \cdot \text { changes }\right) \\
& \wedge \text { unchanged }\left(\left(\mathrm{V}-\mathrm{CH}\left(\mathrm{a}_{\mathrm{i}}\right)\right) \cup \operatorname{PAR} \mathrm{R}_{\mathrm{in}}\left(\mathrm{a}_{\mathrm{i}}\right)\right)
\end{aligned}
$$

In this translation, notice that explicit TLA unchanged predicates are added in order to indicate which variables remain unchanged by the action. Let the notation TLA-action $\left(\mathrm{a}_{i}\right)$ denote the resulting TLA action when a CSL action $\mathrm{a}_{i}$ is translated into TLA. In TLA, the operator Enabled, e.g. Enabled a, is used to denote the fact that the action a is enabled, i.e. it may be executed. Using the approach described above, Enabled TLA-action $\left(\mathrm{a}_{\mathrm{i}}\right)$ corresponds to $\mathrm{a}_{\mathrm{i}}$.enabled . In addition, when $a_{\mathbf{i}}$.defined is not satisfied, any state can be considered as a next state.

With respect to the behavior of an object, TLA and CSL have the same semantics, i.e. the initial conditions hold first and then either an action is executed or the specification variables remain unchanged. Therefore, the translation is one to one for the object behavior.

Once the CSL actions are translated into TLA, abstract events can also be translated into TLA. Abstract events are represented by the conjunction of the involved actions and the constraint of the abstract event. Local variables of an abstract event are introduced using TLA temporal existential quantification $\exists$ which denotes hiding of variables in TLA. Hiding of abstract events in a composition is translated by hiding the parameters of the actions involved in these abstract events.

Specialization in TLA is based on the existence of a refinement mapping between the specifications. It is general enough to cover CSL specialization. In other words, let TLA-spec(M) denote the CSL specification M translated into TLA, if the CSL specifications $M$ and $N$ are such that $N$ is a specialization of $M$, then TLA-spec(N) $\Rightarrow$ TLA-spec(M).

In CSL, we have a composition rule which can be used to show that the composition of a given number of specifications is a specialization of another higherlevel specification. The intuition behind this composition rule is: given a CSL composition of $\mathrm{M}_{1}$ and $\mathrm{M}_{2}$, if

1) we have internal consistency in that composition which means that each component behaves well in an environment including the other components with respect to the abstract events relating the latter components to the former;

2) and the composition, considering visible the actions of the components and the abstract events which remain, is a specialization of another high-level specification $M$ then the composition of $M_{1}$ and $M_{2}$ is a specialization of $M$.

Once internal consistency in a composition is shown, specialization suffices to have a sound composition rule. Internal consistency can be demonstrated by showing that none of the abstract events has a participating action for which the "defined" predicate is not satisfied. Once the CSL specifications are translated into TLA, it can be 
demonstrated as an invariant of the resulting TLA specification. This is achieved using the TLA rule INV1 [6]. As explained, specialization is proven at the level of the TLA specifications. This has for consequence that CSL compositional reasoning can be achieved at the level of the resulting TLA specification using TLA rules.

On the other hand, to reason about specifications which include explicit assumptions about the environment, TLA introduces the operator $\pm_{>}[1]$. A specification $E \pm_{>} M$ is a specification of an open system where $E$ denotes the assumptions about the behavior of the environment and $M$ denotes the behavior of the component. In CSL, the assumptions about the environment are at the level of actions, while in TLA, $E \pm_{>} M$ specifications are introduced to take into account the assumptions about the environment at the level of the whole specification. This means undefined situations are dealt at the level of the whole specification, i.e. undefined situations have coarser granularity in TLA $E \pm_{>} M$ specifications. One may suggest that TLA E $\pm_{>} M$ specifications should be comparable with the specifications obtained by translating CSL specifications into TLA. However, E $\pm_{>} M$ specifications require explicit synchronization between the environment and the module actions. This synchronization is achieved by sharing variables. This contrasts with the fact that CSL action synchronization is translated into action conjunction, resulting in a more abstract specification. $E_{>} M$ specifications, because of the explicit synchronization through shared variables, have an implementation bias.

\subsection{Developmental approach and Tools}

The developmental approach which is used in this case study proceeds as follows. First the structure model is built. It describes the application objects and the associations between them including aggregations. The notation which is used is the one proposed in [10]. In the structure model, the different associations may have application-specific behavior and behavioral constraints as described in [10]. These allow to identify the actions of the objects participating in the associations and the interactions between these objects in terms of abstract events. The description of these interactions is achieved in the interaction model.

Once the structure and interaction models are built, we then use CSL to describe in more detail the objects, composite objects, actions of these objects, and the interactions in terms of abstract events. As noted earlier, XOMT follows closely the OMT rules. In OMT, the behavior of objects is described by means of statecharts [3]. By adopting CSL as a vehicle for specifying object behavior, interactions, and compositions, we should say how the computational model of CSL compares to statecharts. CSL describes the behavior of an object by its initial conditions, the invariants, and its actions. The initial conditions represent the starting state in a statechart. An action can be interpreted as a combination of OMT events and the operations performed when the event occurs.

In OMT, events are atomic and they can be specialized. In CSL, actions are atomic and they can be specialized. However, in OMT events may denote an elapsed time. This is not actually handled in CSL, although it can be achieved by timing the action executions. The OMT event trace diagram corresponds to the traces allowed by an object. They can be derived from its CSL specification.

In CSL, states are implicitly captured by the values allowed for the local variables. The organization of states into disjoint substates or composite states can be achieved through predicates on the values of the local variables. It can be demonstrated that a substate inherits the properties of its superstate. However, composite states can only 
be specified in the context of compositions. For instance, the initial conditions of a composition may imply the initial conditions of its components. The initial state of the composition is a composite state bundling the initial states of its components.

In CSL, concurrency is based on interleaving, i.e. at the level of a single object there is no concurrency. When considering a composition, the abstract events occurring in the composite are interleaved with its actions. In an abstract event, more than two components may simultaneously interact. This means, there is concurrency between the interacting components. OMT assumes that an atomic object is a finite state machine with a queue for incoming events. Composite objects may have more queues of events corresponding to each of its components. This contrasts with CSL where even if an object is a sequential system (or process), it has no queue. It accepts or blocks the execution of an action. In addition, it may display an undefined behavior or undergo non-deterministic changes by executing alone one of its actions.

OMT assumes that objects communicate by sending events. In addition, they can interact implicitly if one object has a guard condition that depends on the state of another object, such as being in a given state. Our experience with XOMT has shown that describing synchronous interactions with such a model is cumbersome. In CSL, interaction is based on the concept of abstract event [2]. Abstract events are only meaningful in the context of an object association. This has the consequence that the structure of an application shapes its dynamic behavior.

Apart from events, in a statechart, there are transitions. They correspond to the changes predicates defined in the semantics of actions in CSL. The so-called $\lambda$ transitions in OMT correspond to the non-deterministic changes allowed by an object in CSL.

As a rule of thumb, the interaction specification is done when we may easily determine the enabling and defined states of the objects involved in each abstract event. If we can not determine the enabling and definedness of states of objects involved in the abstract events, we may not determine which abstract events do occur. After providing the CSL specifications, they are translated into TLA formulas. With the TLA specifications which are obtained, the specifier may now use different TLA tools to verify the specifications. Results of this process can be reflected directly into CSL specifications.

\section{Case Study}

As an application of this case study, we have selected the lift problem which is one of the problems proposed for demonstrating the adequacy of a specification method for complex systems. We omit certain details which do not contribute to the essential points of this paper.

\subsection{Problem statement}

A lift system is to be installed in a building with $\mathrm{m}$ floors. It is aimed at moving people from one floor to another. It is used under the following constraints:

1. Each lift has a set of buttons, one for each floor. These illuminate when pressed and cause the lift to visit the corresponding floor. The illumination is canceled when the corresponding floor is visited by the lift.

2. Each floor has two buttons (except for ground and top floors), one to request an up-lift and one to request a down-lift. These buttons illuminate when pressed. The 
illumination is canceled when a lift visits the floor and is either moving in the desired direction, or has no outstanding requests.

3. When a lift has no requests to service, it should remain at its final destination with its doors closed and await further requests.

4. All requests for lifts from floors must be serviced eventually, with floors given equal priority.

5. All requests for floors within lifts must be serviced eventually, with floors being serviced sequentially in the direction of travel.

6. Each lift has an emergency button which, when pressed causes a warning signal to be sent to the site manager. The lift is then deemed "out of service". Each lift has a mechanism to cancel its "out of service" status.

7. The doors shall be closed when the lift moves.

8. The lift is stopped when it reaches the "out of service" state. Furthermore requests made from the lift carriage are then cleared, and no new requests from the lift carriage are accepted.

Many requirements of a lift are not mentioned, since the designer is expected to know what a lift is.

\subsection{Developing the XOMT specification}

3.2.1. Structure model. The structure model is about modeling the associations between objects as well as the structure of these objects in terms of other objects. We use the following rule of thumb for establishing associations between objects. We are justified in establishing an association between two objects, A and B, if and only if we want to express structural or behavioral constraints between the two objects. On the other hand, the structure of objects depend on the specifier and the level of detail that is required for the model.

We first provide an high-level structure model of a building since the user, the lift and the floors are in the context of a building. In structure models, objects are represented by rectangles and aggregation is shown by embedding one object into another. The structure model for the building can be interpreted as follows. We have a building which is a composite object consisting of Floor and Lift objects. When necessary the cardinality of the components are shown in a structure model. The object associations are shown in a structure model. Floor objects are associated between them. Each Floor is associated to the Lift through several associations.

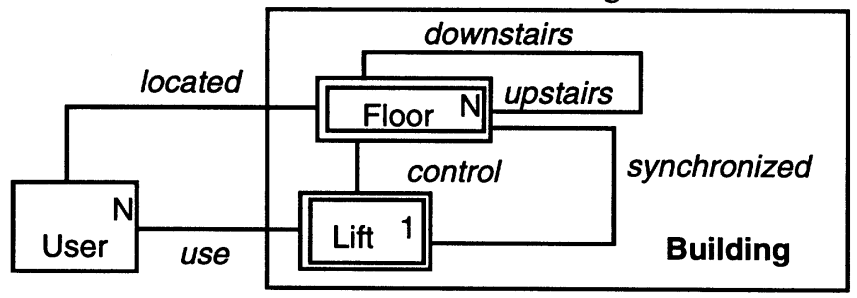

Figure 2. High level structure model of the lift application.

The main object associations abstract the constrainment between the floor and the lift. When a user pushes the button at a floor, this propagates through the association "control" linking that floor to the lift. In addition, when the lift arrives at a given floor, if it stops and opens its doors, the floor doors must also be opened. This is captured by the association "synchronized". A user may be located at a given floor or traveling through the building using the lift. These information are respectively captured by the associations "located" and "use". 
After providing this high-level view, we provide more details by describing the Lift object. As a rule of thumb, we use one structure model per composition when the composition is complex enough, or we regroup several compositions in a single structure model. In the structure model below, the Lift and its components are detailed. Please do not confuse Floor(i) with Floor objects, the former represent buttons inside the Lift while the latter is the real Floor.

In the structure model of the application, illustrated in Figure 2, the lift being a visible component of the building, it follows that the visible components of the lift are also visible at the level of the building. A lift has a ControlPanel and doors. The ControlPanel has various buttons including two buttons which control the opening and closing of the lift doors.

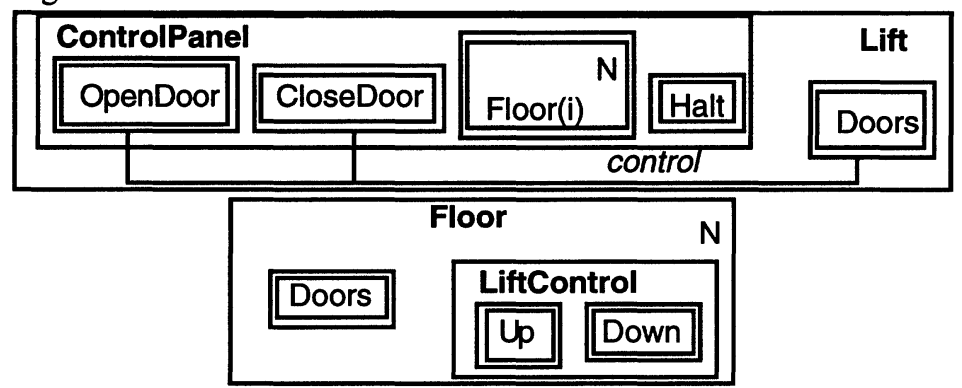

Figure 3. Detailed structure model of the lift and the floor.

This is followed by a detailed structure model of the Floor. Each Floor object consists of Doors and a LiftControl. The LiftControl is an abstraction for the two buttons which control the Lift at each Floor. Notice that in order to avoid too much details on this structure model, we have left out the case of the first and the last Floors which have only one button per LiftControl. However, this can be captured by making the components of the LiftControl optional.

The structure models describe one lift per building. In a multi-lifts building we may have considered an additional object responsible for the coordination of the lifts. Such an object may be called a LiftManager.

3.1.2. Interaction specification. An interaction specification portrays the interactions between the objects forming the application. These interactions are described in terms of abstract events involving the objects of the applications. These abstract events occur only in the context of object associations. The associations which embed abstract events include but are not limited to the following associations: (1) between lift doors and floor doors, (2) between up button and the lift, (3) between down button and the lift, (4) between floor(i) button and the lift, (5) between openDoor button and the lift, (6) between closeDoor button and the lift, (7) between halt button and the lift, (8) user and the lift.

The association 1 corresponds to "synchronized"; 2 and 3 correspond to "control"; 4, 5,6, and 7 correspond to the associations within the Lift composition; finally, the association 8 corresponds to "use". The abstract events are described based on the requirements and assumptions below:

Lift and the doors

- When the lift arrives at a given floor where the button is pressed and the lift is going toward the direction indicated by the button, this makes the lift stop at this floor and open its doors. 
- When the lift arrives at a given floor which was a target direction, this makes the lift stop and open its doors.

- When the lift is stopped and the button open door is pressed, this makes the lift to open its doors.

- When the lift doors are opened (respectively closed), the corresponding floor doors are also opened (respectively closed).

Up and down buttons

- When the lift arrives at a given floor which was a target direction, and the button up or down is pressed, these buttons should be deactivated. If both are pressed, nondeterministic choice is applied to deactivate only one button.

- When the lift arrives at a given floor which is in the direction of the target floor, and the button leading to the target floor is pressed, this makes the button to be deactivated.

Control panel

- When floor(i) button is pressed, this causes the indicated floor to be a target destination.

- When the button open (close) door is pressed if the lift is stopped, this makes the lift open (close) its doors.

- When the button halt is pressed, this leads the lift to the nearest floor and stops the lift at that floor canceling all the target destinations.

To make the description of the behavior of the lift tractable, we have decomposed its movement into two specific discrete steps, namely move(i,j) and stop(k). move(i,j) models the displacement from the floor $i$ to the floor $j$. It is achieved in one complete execution step and it is atomic. stop(k) models the action forcing the lift to stay at the floor $\mathrm{k}$.

In the following, we describe a simplified Lift system which can be later refined to include all the requirements. It includes a button, the lift, the user and the doors specifications. A button is described by the following CSL specification :

spec Button(direction:Direction, floor:FloorNumber)

define ButtonState $=\{$ idle,on $\}$

define Direction $=$ none, up, down $\}$

local variables

state : ButtonState; serviced : Boolean

initial conditions

behavior state $=$ idle $\wedge$ serviced $=$ false

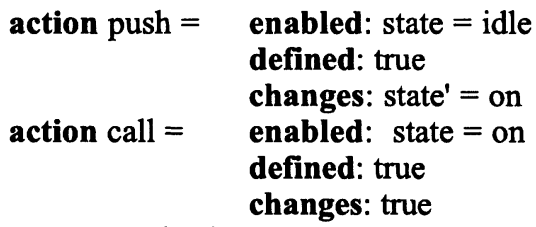

action serviced $=$

enabled: state $=$ on

defined: (serviced=false)

changes: (serviced'= true)

action release $=$ enabled: $($ state $=$ on $) \wedge($ serviced $=$ true $)$

defined: true

end spec Button

changes: $\left(\right.$ state $^{\prime}=$ idle $) \wedge($ serviced' $=$ false $)$

The specification is parameterized to allow the generic specification of buttons according to the direction and the floor which are serviced by the button. The actions of the button can be described informally as follows. The push action consists to 
press the button. call is an action by which the button communicates with the lift. serviced is an action by which the lift notifies the button that it has stopped at the corresponding floor.

Based on the semantics of CSL actions introduced in Section 2, there are many specification variants for an action. For instance consider the push action. It can be specified as:

action push $=$ enabled: true

defined: true

changes: state' $^{=}$on

The action is always enabled and it is never results in an undefined behavior.

action push $=$ enabled: state $=$ idle

defined: true

changes: state' $^{=}$on

The action is enabled in states where the button is idle and it is always welldefined. In other words, you may call the action only when the button is idle.

action push $=$ enabled: true

defined: state $=$ idle

changes: state' $^{=}$on

The action is always enabled. It results in undefined behavior in states where the button is on.

action push $=$ enabled: state $=$ idle

defined: state $=$ idle

changes: state' $=$ on

The action blocks in states where the button is on and it never results in undefined behavior.

It is up to the specifier to select the intended behavior. Actually, the first specification of the action push is the one which corresponds to the reality since we are allowed to push the button independently of its state.

In the requirements, we have stated that buttons are illuminated. This is specified by having a light in each button. The light object is described below.

spec Light

local variables

lit : Boolean

initial conditions

behavior lit $=$ false

action illuminate $=$

action deilluminate $=$

enabled: lit $=$ false

defined: true

changes: lit' $=$ true

enabled: lit $=$ true

defined: true

end spec Light

changes: lit' $=$ false

The specification of a button which has a light is as follows :

spec ButtonWithLight

extends Button

contains

invariants

light : Light

light.lit $=($ state $=$ "on" $)$

end spec ButtonWithLight 
The invariant has to be satified by any implementation of this specification. In fact, there should be a relation between the implementation of the invariant and the two actions provided by the specification Light. In the remaining of this paper, we no longer describe the inner-workings of actions in terms of enabled, defined and changes predicates.

We assume the operators : first_floor() returning the first floor of the building, last_floor() returning the last floor of the building, and next floor(direction, $f, n$ ) returning the floor following a given floor based on the direction. Direction can be none, up, and down.

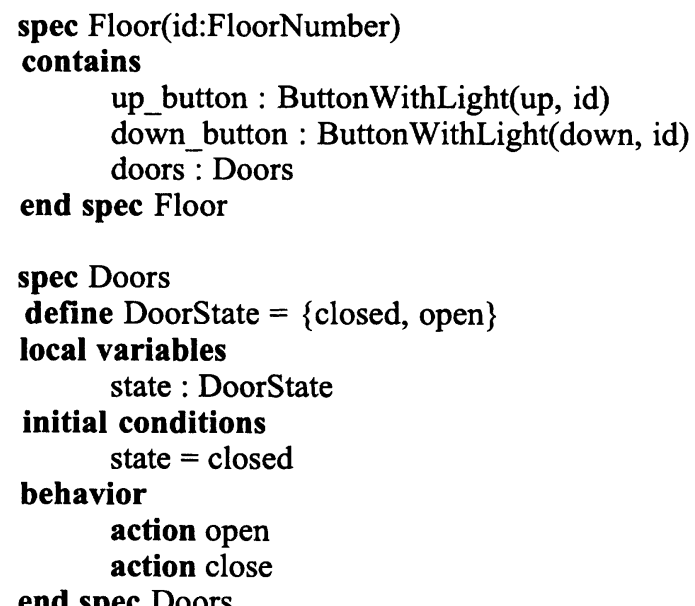

end spec Doors

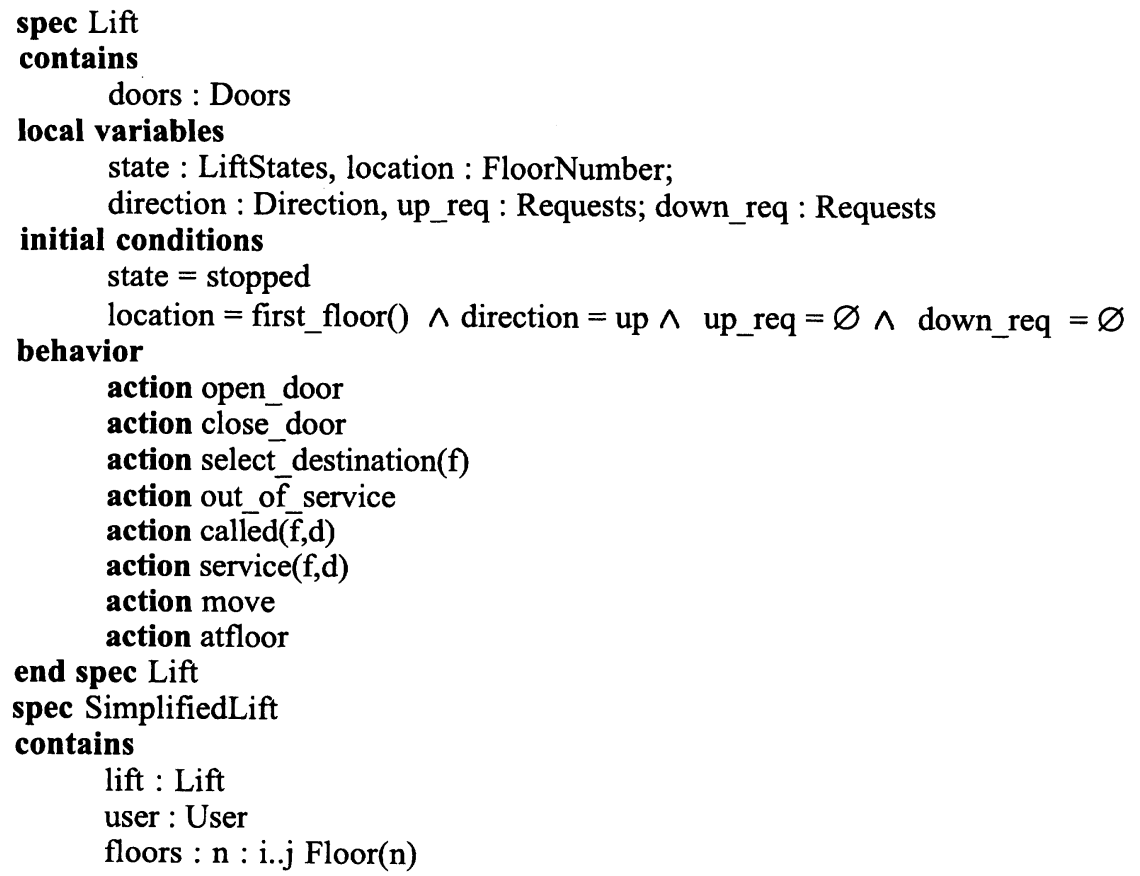


abstract events

association control

$$
\begin{aligned}
& \text { event } \text { callup }=\text { actions: floors[n].up_button.call } \\
& \text { lift.called(f, d) } \\
& \text { constraint: }(f=\text { floors[n].up_button.floor) } \wedge \\
& \text { ( } d=\text { floors[n].up_button.direction) } \\
& \text { event calldown }=\text { actions: floors }[n] \text {.down_button.call } \\
& \text { lift.called(f, d) } \\
& \text { constraint: }(f=\text { floors[n].down_button.floor }) \wedge \\
& \text { ( } d \text { = floors[n].down_button.direction) }
\end{aligned}
$$

... the other abstract events are defined similarly

end spec SimplifiedLift

\subsection{Developing the formal specification}

In the following, we only portrays the translation of Button and Light specifications.

3.3.1. TLA Specifications. In the TLA specifications below, each CSL specification is mapped into a module. An extends clause corresponds to the TLA extends clause. A contains clause is mapped into instantiation of the modules corresponding to the components. Local variables are introduced as parameters of the module. At this point, the translation becomes one to one since the initial conditions clause is translated into an Init predicate, and the actions are translated based on the approach sketched in Section 2. They are included in the temporal section of the module. For the CSL specification Light, $\mathrm{V}=\{$ lit $\}, \mathrm{LV}$ (illuminate $)=\{$ lit $\}, \mathrm{LV}$ (deilluminate $)=$ $\{$ lit $\}, \operatorname{PAR}($ illuminate $)=\varnothing$, and $\operatorname{PAR}($ deilluminate $)=\varnothing$. This leads to the following translation :

\section{Module Light}

parameters

predicates

$$
\text { lit : Variable }
$$

actions

$$
\text { Init } \equiv \text { lit }=\text { false }
$$

$$
\begin{aligned}
& \text { illuminate } \equiv \wedge(\text { lit }=\text { false }) \wedge(\text { true } \Rightarrow \text { lit' }=\text { true }) \\
& \text { deilluminate } \equiv \wedge(\text { lit }=\text { false }) \wedge(\text { true } \Rightarrow \text { lit' }=\text { true })
\end{aligned}
$$

temporal

Actions $\equiv$ illuminate $\vee$ deilluminate

Behavior $\equiv$ Init $\wedge \square[$ Actions $]<$ bulb $>$

In the translation of the CSL specification Button, we have taken care of adding extra predicates state $\in$ ButtonState and floor $\in$ FloorNumber in order to reflect the typing of the local variables in the TLA specification.

parameters

$$
\text { Module Button }
$$

direction : Variable

floor : Variable

state : Variable

serviced : Variable

predicates

calling: Variable

state $\in$ ButtonState $\wedge$ floor $\in$ FloorNumber 


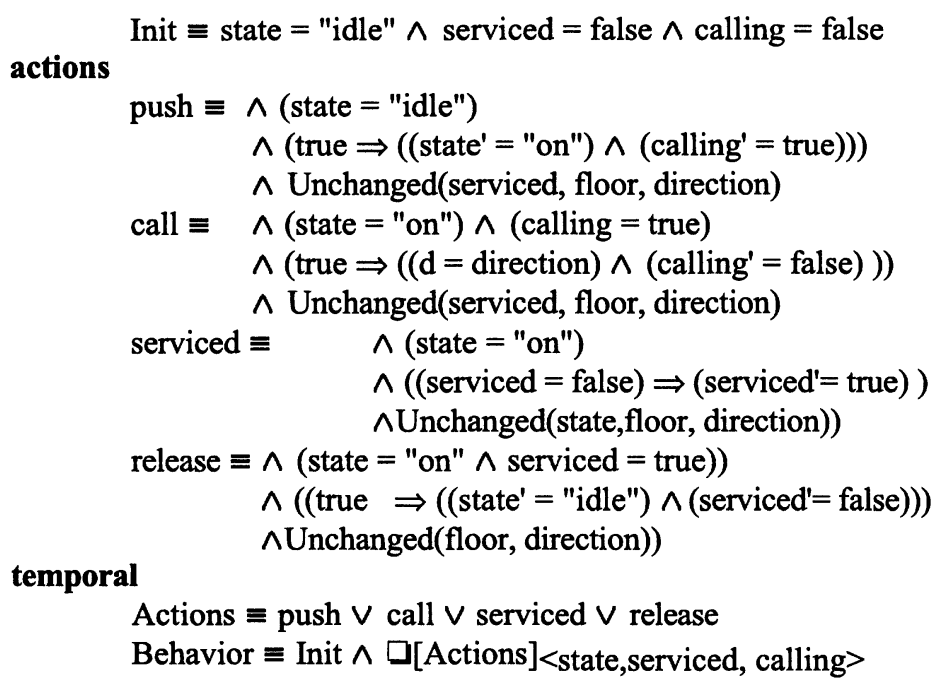

An abstract event is translated using conjunction of actions. For instance, the abstract event callup in the specification SimplifiedLift is translated into the following TLA action :

callup $\equiv \exists \mathrm{f}$ : FloorNumber, d: Direction , $\mathrm{n} \in\{\mathrm{i}, \mathrm{i}+1, . ., \mathrm{j}\}$ :

$$
\begin{aligned}
& \wedge \text { floors[n].up_button.call } \\
& \wedge \text { lift.called(f, } d) \\
& \wedge(\wedge(f=\text { floors[n].up_button.floor }) \\
& \quad \wedge(d=\text { floors[n].up_button.direction) })
\end{aligned}
$$

Based on the TLA specifications, various properties of the lift system may be verified.

3.3.2. Proving various properties. For the lift system, deadlock freedom can be interpreted as the conjunction of two safety properties:

a) In the initial state at least one abstract event (represented by a TLA action) is enabled.

b) Each abstract event leads the objects into a state where at least one abstract event is enabled.

In order to prove this property, we use the TLA INV1 rule shown below [6].

$$
I \wedge[\mathrm{N}]_{f} \Rightarrow I^{\prime}
$$

$$
I \wedge \square[\mathrm{N}]_{f} \Rightarrow \square I
$$

This rule is used to prove that a program satisfies an invariance property $\square I$. The hypothesis asserts that a $[\mathrm{N}]_{f}$ step cannot falsify $I$. The conclusion asserts the if $I$ is true initially and every step is a $[\mathrm{N}] f$ step, then $I$ is always true.

In our case, $I$ represents the disjunction of the enabling conditions of the actions provided by the program. It suffices to choose the invariant property $I$ as at least one abstract event is enabled to prove the deadlock freedom property using the above rule. For the SimplifiedLift, this can be expressed as the following TLA formula 
Notice that (Enabled callup) can be reduced to the conjunction of Enabled floors[n].up_button.call, Enabled lift.called(f,d), and ( $\mathrm{f}=$ floors[n].up_button.floor) $\wedge(\mathrm{d}=$ floors[n].up_button.direction) $)$ by predicate logic since an abstract event is enabled when all its constituant actions are enabled and its constraint is true.

Liveness properties such as "when the button of a floor is pressed the lift will eventually stop at this floor" are proved using TLA's WF1 or SF1 rules described in [6]. To ease the proof of this property, we decompose the property into two properties denoted by a) and b) below. The WF1 rule is used to prove properties of the form $\mathrm{P}$ leads-to $\mathrm{Q}$ from a weak fairness condition $\mathrm{WF}_{f}(A)$. The reader is referred to [6] for more details on weak fairness conditions for TLA actions. Here $A$ denotes a specific action. An $A$ step is understood as the execution of the action $A$. It can be applied when an $A$ step that starts with $\mathrm{P}$ true makes $\mathrm{Q}$ true. The WF1 rule is as follows:

$$
\begin{aligned}
& \mathrm{P} \wedge[\mathrm{N}] f \Rightarrow\left(\mathrm{P}^{\prime} \vee \mathrm{Q}^{\prime}\right) \\
& \mathrm{P} \wedge\langle\mathrm{N} \wedge A\rangle_{f} \Rightarrow \mathrm{Q}^{\prime} \\
& \mathrm{P} \Rightarrow \text { Enabled }\langle A\rangle_{f}
\end{aligned}
$$

$\square[\mathrm{N}]_{f} \wedge \mathrm{WF}_{f}(A) \Rightarrow \mathrm{P}$ leads-to $\mathrm{Q}$

The two liveness properties $\mathrm{a}$ ) and $\mathrm{b}$ ) are proven using the following assumptions: a) When we press the button of a floor then the floor number will eventually be listed in the request list of the lift: This property, i.e. $\exists \mathrm{f}$ : Floor : P leads-to Q, is proven with TLA WF1 by taking $P$ as

(floors[n].up_button.state $=$ "on" $\wedge f=$ floors[n].up_button.floor)

$\vee$ (floors[n].down_button.state $=$ "on" $\wedge \mathrm{f}=$ floors[n].down_button.floor)

$\mathrm{Q}$ as

$$
f \in \text { lift.up_req } \vee f \in \text { lift.down_req }
$$

and $A$ as the abstract event callup or calldown, respectively, where: callup $\equiv \exists \mathrm{f}$ : FloorNumber, d: Direction , $\mathrm{n} \in\{\mathrm{i}, \mathrm{i}+1, \ldots, \mathrm{j}\}$ :

$\wedge$ floors[n].up_button.call

$\wedge$ lift.called(f, $\mathrm{d})$

$\wedge(\wedge(f=$ floors[n].up_button.floor $)$

$\wedge(d=$ floors[n].up_button.direction $))$

calldown $\equiv \exists$ f: FloorNumber, d: Direction , $n \in\{i, i+1, . ., j\}$ :

$\wedge$ floors[n].down_button.call

$\wedge$ lift.called(f, $d)$

$\wedge\left(\wedge\left(f=\right.\right.$ floors$\left.[n] . d o w n \_b u t t o n . f l o o r\right)$

$\wedge(\mathrm{d}=$ floors$[\mathrm{n}]$.down_button.direction $))$

WF $f(A)$ is deduced from the fairness of floors[n].up_button.call, floors[n].down_button.call, and lift.call actions. In the SimplifiedLift specification, by default the TLA actions are supposed to have weak fairness.

b) If the floor number is listed in one of the request lists of the lift then the lift will stop at the corresponding floor: This property is internal to the lift and it can be deduced from the sequence of states of the lift module, i.e. its behavior. It can be also broken down into smaller properties.

Proofs are tedious and complicated. A great deal of these proofs can be mechanical and take advantage of the structure of the formulas to decompose the proofs. There are many tools which can assist in proving the properties of TLA specifications. At the 
University of Dortmund, a certain number of tools for developing, preparing, building, testing and verifying TLA specifications have been prototyped [7]. Among these tools, we selected eTLA+ which is an interpreter allowing the interpretation of specifications combined with graphical visualization of their execution. Once the CSL specifications are translated into TLA, TLA specifications can then be translated into eTLA+ which is used as input for the eTLA+ interpreter. The eTLA+ interpreter allows symbolic debugging including stepwise or continuous execution and tracing. Non-determinism is handled by allowing the user to select the next action to execute or if he may prefer a scheduling strategy. CSL specifications can also be translated in TLALight, another variant of TLA [7]. TLALight specifications are implemented using a $\mathrm{C}++$ translator which derives a distributed implementation prototype as a set of communicating processes in a workstation network.

\section{Conclusion}

In this paper, we have modified the developmental approach used in XOMT in order to integrate a new approach to the description of composite object behaviors. XOMT has now two distinct views which portray the structure and the dynamic behavior of applications. The developmental approach is compositional since we can connect different pieces of the design by establishing associations between objects of these pieces. In addition, the approach can be used for component-based systems.

Throughout this case study, the structure of composite objects serves for describing the dynamic behavior of these objects. There is an implicit relation between the CSL formal specifications and the XOMT structure specifications (structure models in XOMT). The characteristics of component associations are such that their presence simplifies proofs of behavioral properties, as compared to general associations. In addition, we can derive useful properties of the composition based on that of its components. For instance, specification invariants of the SimplifiedLift imply properties of its components. This is due to the encapsulation of the local variables in the components. Once the Deadlock freedom of components proved individually, there is no need to prove this again when the components are incorporated in the SimplifiedLift specification, i.e. the proof of deadlock freedom for the SimplifiedLift is limited to the actions and the abstract events defined in that specification. Furthermore, based on the interconnections between components, we may prove progress properties for the SimplifiedLift. This is illustrated with the proof of the property "when we press the button of a floor then the floor number will eventually be listed in the request list of the lift".

In addition, the approach proposed in this paper is based on the linkage between the structure and the behavior of composite objects. Such an approach was shown to be adequate when dealing with complex systems since the complexity of systems is concentrated on the interactions between components of such a system. CSL specifications can be translated into TLA, for which tools can be used for verification purposes as well as for checking the composition of different pieces of the design.

In this paper, we sketched how different tools can be combined for supporting the XOMT developmental approach. In order to support this development process, the adaptation of a case tool, namely MetaEdit tool [8] is underway. The MetaEdit tool supports various object notations and methods, in addition it is parameterizable in the sense that you may define your own notation and method by defining a meta model of the notation. The MetaEdit tool views an object-oriented method as a set of notations and specifications constructed using these notations. We use this latter feature for defining a meta model for XOMT. 
Future development of this work includes the design and the implementation of a tool supporting CSL with an integrated environment for verifying the specifications. This may require the design and implementation of an automatic translator of CSL specifications to TLA specifications.

\section{References}

[1] Abadi, M., Lamport, L., Conjoining Specifications, ACM TOPLAS , November 1995.

[2] Bochmann, G.v., Abstract dynamic modelling of complex systems, Publication départementale No 863, Dépt. IRO, Université de Montréal, Janvier 1993.

[3] Harel, D., Statecharts: a Visual Formalism for Complex Systems, Science of Computer Programming, Vol. 8, No. 3, pp.231-274, 1988.

[4] ISO - Information Processing Systems - Open System Interconnection, LOTOS - A Formal Description Technique Based on The Temporal Ordering of Observational Behaviour, DIS 8807, 1987.

[5] Järvinen et al., Object-Oriented Specification of Reactive Systems, Proc. of 12 th ICSE, March 1990, IEEE Computer Society Press, p. 63-71.

[6] Lamport, L., The Temporal logic of actions, ACM TOPLAS 16(3):872-923, May 1994.

[7] Mester, A., Herrmann, P., Tools for TLA-based specifications, RvS-TLA-94-35, February 1994, University of Dortmund.

[8] MetaEdit Personal 1.2, Customisable Case Tool to meet your requirements, MetaCase Consulting, Finland, 1995.

[9] Ramazani, D., Contribution of Object-Oriented Methodologies to the Specification of Complex Systems, Proceedings of Fifth Complex Systems Engineering Synthesis and Assessment Technology Workshop (CSESAW'95).

[10] Ramazani, D., Bochmann, G.v., Extending Object Modelling Technique for the Specification of Composite Objects, In Proceedings of TOOLS-USA96, July 1996.

[11] Ramazani, D., Bochmann, G.v., Approaches to the Specification of Object Associations, In Proceedings of FMOODS97, July 1997.

[12] Rumbaugh, J., Blaha, M., Premerlani, W., Eddy, F., Lorensen, W., Object-Oriented Modeling and Design, Prentice-Hall, Englewood Cliffs, NJ, 1991. 